\title{
On the radio emission of the preflare active regions
}

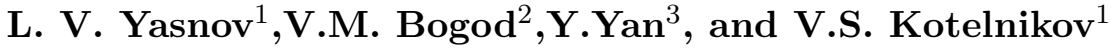 \\ ${ }^{1}$ Radiophysics Research Institute, Saint-Petersburg State University,Saint-Petersburg, Russia \\ email: Leonid.Yasnov@paloma.spbu.ru \\ ${ }^{2}$ Special Astrophysical Observatory RAS, Saint-Petersburg, Russia email: vbog@gao.spb.ru \\ ${ }^{3}$ National Astronomical Observatories CAS, Beijing, China email: yyh@bao.ac.cn
}

This work is devoted to the new phenomena appearing in radio emission in preflare active regions that were found with the use of radio telescope RATAN-600. The first is the complicated spectral-polarization behavior in narrow frequency range and, in particularly, the double changing of the polarization emission sign in active region at the time interval of several days before big flare. The second, the detection of regular decreasing of radio brightness radio darkening of active regions, which is also observed in a few days prior to flare [Tokhchukova \& Bogod (2003)].

We have considered several mechanisms that can result in double change of the polarization sign. It was shown that the mechanism of linear interaction of waves in the region of a quasitransversal magnetic field cannot give an observed picture of this type of polarization inversion. It is easy to obtain an observed picture of change of a polarization sign with the propagation of waves through region of a zero magnetic field, but only with severe demands on geometry of the region of a zero magnetic field. It would be difficult to expect, that these conditions can be met frequently in the active regions. In the case of the current layer stabilized by a weak magnetic field orthogonal to a surface of the layer, the opportunities for interpretation of observable change of the polarization sign is get essentially more.

The other version of double change of the polarization sign may be connected with the scattering of radio waves on waves of high-frequency plasma turbulence, which occurs only at a high level of plasma turbulence and result in to the increasing intensity of a radio emission.

We also studied the influence of the filaments on the transmission radio emission. The cold filament does not result in double change of the sign of polarization. In case of the model with the hot filament it is easy to obtain the observed characteristics. Such filament may result in the occurrence of so-called cyclotron lines.

The very important case may be connected with the presence of magnetic "holes", in which the direction of magnetic field lines changes the sign in relation to the observer. It is coincidence with the characteristics observed. Such "holes" really exist in the reconstructed magnetic field of the active region. However, such structure of a magnetic field should be essentially isolated. Otherwise the sign polarization will correspond to direction of a magnetic field in the region of transition from anisotropic to isotropic propagation of radio waves where the structure of a field would not contain any more magnetic "hole". Radio darkening of active region brightness may be connected with the downturn of intensity of a magnetic field at heights of generation of a radio emission. Thus the radio emission reacts to preflare processes in active region noticeably earlier then the photosphere magnetic field. It specifies that preflare processes be more distinctly seen in chromospheres and coronal magnetic fields. 
This work was supported by RFBR ( 02-02-17733 and 02-02-16430)

\section{References}

Tokhchukova, S.Kh. \& Bogod, V.M. 2003 Solar Physics 212, 99. 